\title{
Neurosarcoidosis: Definition and Consensus Diagnostic Criteria
}

\section{Nörosarkoidoz: Tanımı ve Konsensüs Tanı Ölçütleri}

\author{
Istanbul Bakirkoy Prof. Dr. Mazhar Osman Bakirkoy Prof. Dr. Mazhar Osman Psychiatric, Neurologic, and Neurosurgical Diseases \\ Training and Research Hospital, Clinic of Neurology, Istanbul, Turkey
}

Keywords: Neurosarcoidosis, diagnostic criteria, neuroimmunology

Anahtar Kelimeler: Nörosarkoidoz, tanı ölçütleri, nöroimmünoloji

\section{Neurosarcoidosis: Definition and Consensus Diagnostic Criteria}

Sarcoidosis is one of the diseases that often cause diagnostic uncertainty because of different clinical findings. This is especially evident in cases with neurologic involvement because the clinical presentation is very diverse and not specific. The lack of consensus on the diagnosis of neurosarcoidosis has given rise to difficulties in the management of patients and clinical trials.

In the clinical review "Definition and Consensus Diagnostic Criteria for Neurosarcoidosis from the Neurosarcoidosis Consortium Consensus Group” published in JAMA Neurology, diagnostic criteria have been published in order to establish a standardization of the diagnosis of the disease (1). By using the keyword "neurosarcoidosis", a group of 10 neurologists and 5 pulmonologists reviewed publications from PubMed between January 2007 and November 2017.

Non-caseating granulomatous inflammatory reaction in cases of central nervous system involvement was emphasized as a pathologic hallmark and it was mentioned that inflammation tended to be in a perivascular distribution in parenchymal involvement because these granulomas are located at the surface of the brain. The microscopic structure of the granulomas is composed of multinucleate giant cells formed by macrophages and surrounded by lymphocytes and plasma cells. Asteroid bodies, which are another characteristic, but not frequent, histologic feature and stellate-like inclusions within a central vacuole of the giant cell were counted among these findings. It has been pointed out that neuropathologically similar findings may be present in chronic infections and that detailed investigations should be conducted. It has been mentioned that granulomatous inflammatory infiltration, vasculitis, and necrotizing vasculitic findings can be detected in peripheral nervous system involvement.

There is a wide range of clinical findings in terms of the involved region of the nervous system. Findings such as fatigue, cognitive difficulties, and small fiber neuropathy with no evidence of nervous system inflammation were collected under the title of paraneurosarcoidosis and this finding was said to be multifactorial.

In a meta-analysis involving more than 1000 patients, 31\% of patients with neurosarcoidosis had no systemic findings at the beginning, but $84 \%$ of patients were reported to eventually develop other systemic findings during follow-up (2). The importance of directing attention to these sites was emphasized because the lung, intrathoracic lymph nodes, eye, and skin are frequently involved.

It was pointed out that frequent cerebrospinal fluid (CSF) findings included elevated protein and lymphocytic pleocytosis, but that there might be findings that reflect a large number of non-specific inflammatory processes and that infectious screening

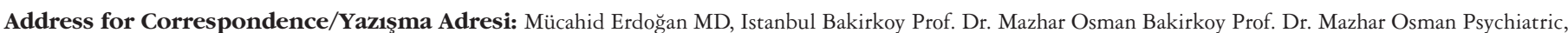
Neurologic, and Neurosurgical Diseases Training and Research Hospital, Clinic of Neurology, Istanbul, Turkey Phone: +90 5302883416 E-mail: erdoganmucahid@gmail.com ORCID ID: orcid.org/0000-0002-4919-602X Received/Geliş Tarihi: 12.02.2019 Accepted/Kabul Tarihi: 23.02.2019

${ }^{\circ}$ Copyright 2019 by Turkish Neurological Society Turkish Journal of Neurology published by Galenos Publishing House.
} 
should be performed for differential diagnosis. CSF and serum angiotensin converting enzyme levels were reported to be controversial due to low sensitivity and specificity. The use of serum lysozyme level is also limited due to low sensitivity and specificity.

A high-resolution chest computed tomographic scan (HRCT), preferably with contrast, when chest X-ray results are normal in the diagnosis of pulmonary sarcoidosis, and fluorodeoxyglucose positron emission tomographic scan (PET) in extrapulmonary involvement have been proposed. Conjunctival biopsy has been proposed to be used in carefully selected patients due to low diagnostic yield.

Cranial and spinal magnetic resonance imaging, CSF analysis, and electromyography, nerve or muscle biopsy, when needed, are recommended for the diagnosis of neurosarcoidosis. In the event of suspected occult disease, comprehensive ocular examination, HRCT, and a whole-body PET scan for the detection of lymphadenopathies are considered appropriate.

Patients who were considered to have neurosarcoidosis in the light of clinical and diagnostic tests in the diagnostic criteria of the disease were classified as possible, probable, and definite neurosarcoidosis according to biopsy results (Table 1).

In conclusion, it was aimed to provide standardization in studies with the help of established diagnostic criteria in order to provide guidance in the diagnosis of neurosarcoidosis presenting with diverse manifestations and in the management of patients. Emphasis was placed on the exclusion of infections and malignancies, and the importance of extensive research. It can be concluded that important information can be obtained in the pathophysiology and course of the disease by acting according to certain criteria in the selection of patients.

Ethics

Peer-review: Internally peer-reviewed.

Financial Disclosure: The authors declared that this study received no financial support.
Table 1. Recommended diagnostic criteria for central and peripheral nervous system neurosarcoidosis are divided into three groups as possible, probable and definite

Recommended diagnostic criteria for central and peripheral nervous system neurosarcoidosis

- Clinical findings and MRI, CSF, and EMG findings are compatible with granulomatous

Possible inflammation of the nervous system and other causes are excluded by detailed investigations. - There is no pathologic confirmation of granulomatous disease.

- Clinical findings and MRI, CSF, and EMG findings are compatible with granulomatous inflammation of the nervous system and other Probable causes are excluded by detailed investigations. - There is confirmation of systemic granulomatous disease consistent with sarcoidosis.

- Clinical findings and MRI, CSF and EMG findings are compatible with granulomatous inflammation of the nervous system and other causes are excluded by detailed investigations.

Definite - Nervous system pathology is consistent with neurosarcoidosis.

Type a: Extraneural sarcoidosis.

Type b: No extraneural sarcoidosis (isolated CNS sarcoidosis).

MRI: Magnetic resonance imaging, CSF: Cerebrospinal fluid, EMG: Electromyography, CNS: Central nervous system

\section{References}

1. Stern BJ, Royal W, Gelfand JM, et al. Definition and Consensus Diagnostic Criteria for Neurosarcoidosis: From the Neurosarcoidosis Consortium Consensus Group. JAMA Neurol 2018;75:1546-1553.

2. Fritz D, van de Beek D, Brouwer MC. Clinical features, treatment and outcome in neurosarcoidosis: systematic review and meta-analysis. BMC Neurol 2016;16:220. 